\title{
Might Nailfold Capillaroscopy Be a "Proxy" for Lung Involvement in Connective Tissue Diseases?
}
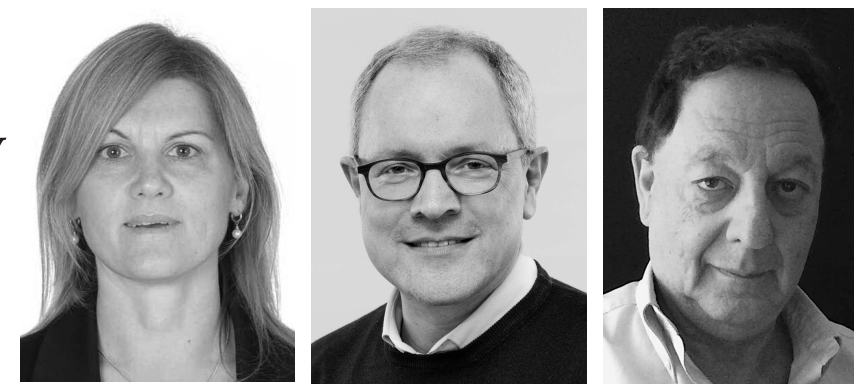

Lung involvement [pulmonary arterial hypertension (PAH)] and/or interstitial lung disease (ILD) are leading causes of death in systemic sclerosis $(\mathrm{SSc})^{1}$. They also contribute significantly to the morbidity and mortality in other connective tissue diseases (CTD), such as Sjögren syndrome (SS), systemic lupus erythematosus (SLE), undifferentiated connective tissue disease (UCTD), mixed connective tissue disease (MCTD), and rheumatoid arthritis (RA) ${ }^{2,3,4}$. Even though no cure is available, timely detection of lung involvement and subsequent administration of appropriate therapies may slow its progression. Nevertheless, a large stumbling block lurks in everyday practice concerning early detection of lung involvement in $\mathrm{CTD}^{5,6}$. More specifically, early detection of lung involvement may be hampered by the nonadherence to guidelines or expert consensus recommendations from PAH or ILD experts 7,8 . For example, the European Society of Cardiology Guidelines suggest screening for PAH in asymptomatic patients with SSc at the initial visit, and expert consensus recommends a high-resolution computed tomography scan (HRCT) at baseline in all patients with SSc to screen for ILD, but a large proportion of the SSc community still does not follow these recommendations in daily clinical practice ${ }^{7,8}$. There may be many reasons for this. One of them is the belief that it is useful to screen only symptomatic patients. Another reason is a lack of real multidisciplinary clinics to care for patients with rare CTD, making it challenging to do specialized investigations such as echocardiography, right heart catheterization, and HRCT. Efforts are being made by different policy makers to address this nonadherence and nonstandardized care. One example is the European Union, which has funded through its health program the European Reference Networks on rare diseases (http://reconnet.ern-net.eu). The aim is to arrange access to equal standardized care for patients with rare CTD throughout Europe, logically with adherence to best clinical practice guidelines ${ }^{9}$. Until good compliance to clinical practice guidelines and expert consensus is achieved, any intermediate help in the best care and cure of patients with CTD is welcome.

Capillaroscopy might be such a supportive aid, because it is a noninvasive tool with easy accessibility, at least in Europe ${ }^{10}$. Historically, capillaroscopy has earned its greatest merits by discriminating patients with a primary Raynaud phenomenon (RP; not related to any condition) from patients with secondary RP (related to a condition) due to SSc, the No. 1 cause of secondary RP in a patient population without additional signs of a $\mathrm{CTD}^{11}$. More specifically, in the last century the seminal papers by Maricq and LeRoy have attested that scleroderma spectrum disorders (SSc, MCTD, dermatomyositis, etc.) are characterized by specific scleroderma patterns on capillaroscopy performed with the wide-field technique (low magnification) ${ }^{12}$. In 2000, Cutolo, et al defined the "early," "active," and "late" SSc patterns using the high magnification technique ${ }^{12 a}$. Since then, several authors have used this subcategorization of scleroderma patterns to associate capillaroscopy changes with organ involvement and disease severity in $\mathrm{SSc}^{13,14,15}$. In addition, various algorithms, based on prospective monocenter or multicenter studies, have been proposed to predict disease progression in $\mathrm{SSc}^{16}$.

In this issue of The Journal, Van Roon, et al assessed the presence of an SSc pattern on nailfold capillaroscopy in consecutive patients with RP at the tertiary referral center, Department of Internal Medicine, University Medical Center Groningen, the Netherlands, and examined its association with pulmonary function tests 2 years or more after presentation ${ }^{17}$. There were 759 patients with RP classified as having primary RP $(n=245)$ when their serology was negative and when they had a "normal" nailfold capillaroscopy and no clinical symptoms of CTD during followup (2 years or more following presentation), or otherwise as secondary RP $(\mathrm{n}=514)$. The latter were subcategorized as

See Capillaroscopy and lung involvement in CTD, page 1109

Personal non-commercial use only. The Journal of Rheumatology Copyright (C) 2019. All rights reserved. 
secondary RP with a definite diagnosis, meeting the classification criteria [SSc, primary SS (pSS), SLE, MCTD, RA], or no definite diagnosis (early SSc based on LeRoy and Medsger ${ }^{17 a}$ criteria, incomplete pSS, incomplete SLE, UCTD). In line with other studies, the SSc pattern on capillaroscopy was present in $88 \%$ of SSc, $33 \%$ of pSS, $17 \%$ of SLE, $71 \%$ of MCTD, and notably in $0 \%$ of all incomplete forms of CTD except (and logically) early SSc according to LeRoy and Medsger ${ }^{17 a}$, where it was present in $97 \%$ of cases. An intriguing novelty of this interesting study was the association with abnormal pulmonary function tests (PFT; a diffusion capacity of the lung for carbon monoxide and/or forced vital capacity $<70 \%$ ) with the SSc pattern on capillaroscopy. Abnormal PFT occurred in 35\% of patients with an SSc pattern (irrespective of an SSc diagnosis), but in only $20 \%$ of patients without an SSc pattern. Binary logistic regression analysis correcting for confounders (such as age, sex, length, weight, and the presence of SSc diagnosis) revealed a negative predictive value (NPV) and a positive predictive value (PPV) of an SSc pattern on nailfold capillaroscopy for predicting abnormal PFT of $70 \%$ and $47 \%$, respectively. When combining positive serology for CTD antibodies (U1-RNP, RNP70, Sm, SSA, SSB, Jo1, topoisomerase, and CENP-B) with SSc pattern on nailfold capillaroscopy, the NPV and PPV increased to $88 \%$ and $46 \%$. The NPV and PPV for abnormal PFT in the secondary RP patients without a definite diagnosis (early SSc, incomplete SLE, incomplete pSS, UCTD, and "other") was $84 \%$ and $30 \%$, respectively, on nailfold capillaroscopy only. For nailfold capillary microscopy and CTD serology combined it was $92 \%$ and $41 \%$, respectively.

Hence, we may accept that performing capillaroscopy in a CTD population may be a supportive aid, concerning CTD-related lung involvement. More specifically, the absence of an SSc pattern in combination with the absence of a CTD antibody may suggest that no lung functional aberrances are imminent. Additionally, the presence of an SSc pattern may indicate possible lung involvement. Nevertheless, the PPV of capillaroscopy alone or combined with a CTD antibody is low $(<50 \%)$, far below what is desirable for a biomarker.

One obvious limitation of this study is that even though it was executed in a tertiary center, there is a relatively high percentage of missing data. Accordingly, echocardiography and HRCT had not been routinely performed. Hence, ideally, rather than focusing on capillaroscopy and CTD antibodies to screen for lung involvement, we may need to screen all our patients with CTD for lung involvement according to clinical practice guidelines and expert consensus. Only in this way would we have all data relevant to lung involvement, allowing the correct holistic management for our patients. We would have multivariate-based algorithms that have sufficient performance characteristics in the prediction of lung involvement. Another limitation of this study is that it contained no patients with a "late" scleroderma pattern, which has been associated with lung involvement in other published literature ${ }^{13,18}$. In addition, some findings concerning capillaroscopic characteristics and lung involvement (e.g., giants, number of capillaries) contrast with the existing literature ${ }^{18,19}$. Hence, we also suggest care in drawing any conclusions concerning what capillaroscopic characteristics within the SSc patterns are associated with lung involvement until systematic reviews on lung involvement and capillaroscopy have been published, with standard interpretation of capillaroscopic characteristics. For now, it is safe to say that the SSc pattern is associated with lung involvement. Of note concerning standardization of interpretation of capillaroscopic characteristics, the European League against Rheumatism Study Group on Microcirculation in Rheumatic Diseases, founded in 2014, has published definitions for standard evaluation of capillaroscopic characteristics ${ }^{20,21,22}$ that may be applied in several rheumatic diseases (www.eular.org/investigative_rheumatology_study_groups.cfm).

Van Roon, et al had the ambitious aim of assessing capillaroscopy as "proxy" for lung involvement in $\mathrm{CTD}^{17}$. The results of their study show that there are signals that both SSc pattern and CTD antibodies are supportive aids in managing patients with CTD. Further efforts from the clinical and scientific community are needed to adhere to good clinical practice guidelines (when existent) in the management of lung involvement in CTD and to collect all relevant data, to produce high-performance algorithms in the early detection of lung involvement in CTD. Capillaroscopy might be incorporated in the development of such algorithms.

\section{ACKNOWLEDGMENT}

Prof. Vanessa Smith is a Senior Clinical Investigator of the Research Foundation - Flanders (Belgium; FWO; 1.8.029.15N). The FWO had no involvement in study design, collection, analysis, or interpretation of data, the writing of the report, or in the decision to submit the article for publication. Special thanks goes to Dr. Amber Vanhaecke for logistic support on this editorial.

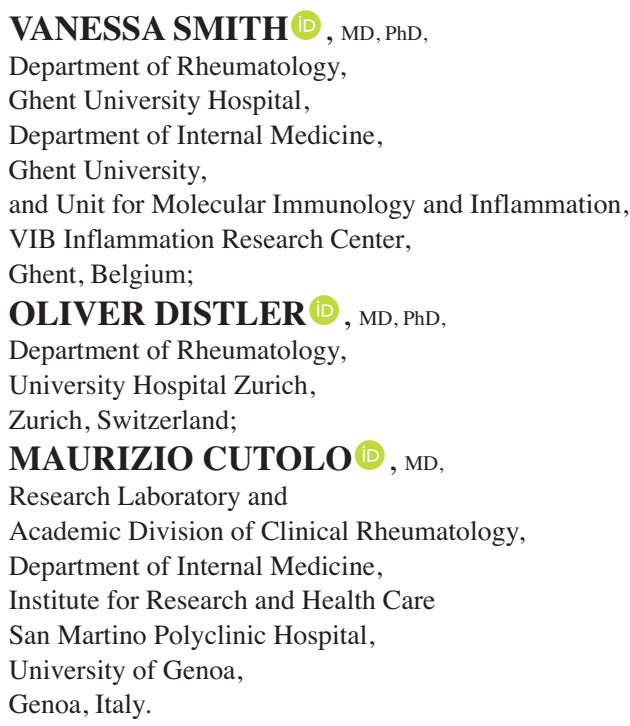


Address correspondence to Dr. V. Smith, Department of Internal Medicine, Ghent University; Department of Rheumatology, Ghent University

Hospital, Corneel Heymanslaan 10,9000 Ghent, Belgium.

E-mail:vanessa.smith@ugent.be

\section{REFERENCES}

1. Elhai M, Meune C, Boubaya M, Avouac J, Hachulla E, Balbir-Gurman A, et al. Mapping and predicting mortality from systemic sclerosis. Ann Rheum Dis 2017;76:1897-905.

2. Trombetta AC, Soldano S, Contini P, Tomatis V, Ruaro B, Paolino S, et al. A circulating cell population showing both M1 and M2 monocyte/macrophage surface markers characterizes systemic sclerosis patients with lung involvement. Respir Res 2018;19:186.

3. Fischer A, West SG, Swigris JJ, Brown KK, du Bois RM. Connective tissue disease-associated interstitial lung disease: a call for clarification. Chest 2010;138:251-6.

4. Raimundo K, Solomon JJ, Olson AL, Kong AM, Cole AL, Fischer A, et al. Rheumatoid arthritis-interstitial lung disease in the United States: prevalence, incidence, and healthcare costs and mortality. J Rheumatol 2018 Nov 15 (E-pub ahead of print).

5. Vandecasteele E, Drieghe B, Melsens K, Thevissen K, De Pauw M, Deschepper E, et al. Screening for pulmonary arterial hypertension in an unselected prospective systemic sclerosis cohort. Eur Respir J 2017;49:5.

6. Wu W, Jordan S, Becker MO, Dobrota R, Maurer B, Fretheim H, et al. Prediction of progression of interstitial lung disease in patients with systemic sclerosis: the SPAR model. Ann Rheum Dis 2018;77:1326-32.

7. Galiè N, Humbert M, Vachiery JL, Gibbs S, Lang I, Torbicki A, et al. 2015 ESC/ERS guidelines for the diagnosis and treatment of pulmonary hypertension: The Joint Task Force for the Diagnosis and Treatment of Pulmonary Hypertension of the European Society of Cardiology (ESC) and the European Respiratory Society (ERS): Endorsed by: Association for European Paediatric and Congenital Cardiology (AEPC), International Society for Heart and Lung Transplantation (ISHLT). Eur Heart J 2016;37:67-119.

8. Khanna D, Strek M, Southern B, Saggar R, Hsu V, Mayes MD, et al. Expert consensus on the screening, treatment, and management of patients with systemic sclerosis-interstitial lung disease, and the potential role of anti-fibrotics in a treatment paradigm for systemic sclerosis-interstitial lung disease: a Delphi consensus study [abstract]. Arthritis Rheumatol 2018;70 Suppl 10:1717.

9. Smith V, Scire CA, Talarico R, Airo P, Alexander T, Allanore Y, et al. Systemic sclerosis: state of the art on clinical practice guidelines. RMD Open 2018;4 Suppl 1:e000782.

10. Ingegnoli F, Ughi N, Dinsdale G, Orenti A, Boracchi P, Allanore Y, et al. An international SUrvey on non-iNvaSive tecHniques to assess the mIcrocirculation in patients with RayNaud's phEnomenon (SUNSHINE survey). Rheumatology Int 2017;37:1879-90.

11. Koenig M, Joyal F, Fritzler MJ, Roussin A, Abrahamowicz M, Boire $\mathrm{G}$, et al. Autoantibodies and microvascular damage are independent predictive factors for the progression of Raynaud's phenomenon to systemic sclerosis: a twenty-year prospective study of 586 patients, with validation of proposed criteria for early systemic sclerosis. Arthritis Rheum 2008;58:3902-12.

12. Maricq HR, LeRoy EC. Patterns of finger capillary abnormalities in connective tissue disease by "wide-field" microscopy. Arthritis Rheum 1973;16:619-28.

12a. Cutolo M, Sulli A, Pizzorni C, Accardo S. Nailfold videocapillaroscopy assessment of microvascular damage in systemic sclerosis. J Rheumatol 2000;27:155-60.

13. Smith V, Decuman S, Sulli A, Bonroy C, Piettte Y, Deschepper E, et al. Do worsening scleroderma capillaroscopic patterns predict future severe organ involvement? A pilot study. Ann Rheum Dis 2012;71:1636-9.

14. Smith V, Riccieri V, Pizzorni C, Decuman S, Deschepper E, Bonroy C, et al. Nailfold capillaroscopy for prediction of novel future severe organ involvement in systemic sclerosis. J Rheumatol 2013;40:2023-8.

15. Ingegnoli F, Ardoino I, Boracchi P, Cutolo M; EUSTAR co-authors. Nailfold capillaroscopy in systemic sclerosis: data from the EULAR scleroderma trials and research (EUSTAR) database. Microvasc Res 2013;89:122-8.

16. Avouac J, Lepri G, Smith V, Toniolo E, Hurabielle C, Vallet A, et al. Sequential nailfold videocapillaroscopy examinations have responsiveness to detect organ progression in systemic sclerosis. Semin Arthritis Rheum 2017;47:86-94.

17. van Roon AM, Huisman CC, van Roon AM, Zhang D, Stel AJ, Smit $\mathrm{AJ}$, et al. Abnormal nailfold capillaroscopy is common in patients with connective tissue disease and associated with abnormal pulmonary function tests. J Rheumatol 2019;46:1109-16.

17a. LeRoy EC, Medsger TA Jr. Criteria for the classification of early systemic sclerosis. J Rheumatol 2001;28:1573-6.

18. Corrado A, Correale M, Mansueto N, Monaco I, Carriero A, Mele A, et al. Nailfold capillaroscopic changes in patients with idiopathic pulmonary arterial hypertension and systemic sclerosis-related pulmonary arterial hypertension. Microvas Res 2017;114:46-51.

19. Hofstee HM, Vonk Noordegraaf A, Voskuyl AE, Dijkmans BA, Postmus PE, Smulders YM, et al. Nailfold capillary density is associated with the presence and severity of pulmonary arterial hypertension in systemic sclerosis. Ann Rheum Dis 2009;68:191-5.

20. Smith V, Beeckman S, Herrick AL, Decuman S, Deschepper E, De Keyser F, et al. An EULAR study group pilot study on reliability of simple capillaroscopic definitions to describe capillary morphology in rheumatic diseases. Rheumatology 2016;55:883-90.

21. Cutolo M, Melsens K, Herrick AL, Foeldvari I, Deschepper E, De Keyser F, et al. Reliability of simple capillaroscopic definitions in describing capillary morphology in rheumatic diseases. Rheumatology 2018;57:757-9.

22. Cutolo M, Melsens K, Wijnant S, Ingegnoli F, Thevissen K, De Keyser F, et al. Nailfold capillaroscopy in systemic lupus erythematosus: A systematic review and critical appraisal. Autoimmun Rev 2018;17:344-52.

J Rheumatol 2019;46:1061-3; doi:10.3899/jrheum.181408 